\title{
Polymeric Matrices for Neural Graft Transplantation
}

\author{
S. Woerly ${ }^{1}$ and D.J. Morassutti ${ }^{2}$ \\ ${ }^{1}$ Institute of Macromolecular Chemistry, Czechoslovak Academy of Sciences, 16206 Prague 6, \\ Czechoslovakia, ${ }^{2}$ Departments of Biology and Neurosurgery, University of Ottawa, Ottawa, Canada
}

One of the main issues in neural grafting technology is the optimization of graft survival and integration into the host brain, prerequisites for the establishment of a functional host-graft relationship. Optimal integration of the transplanted cells is the result of axonal growth from the transplant and the regeneration of axons from host neurons damaged during the transplantation procedure. Glial cells at the graft-host interface should not be allowed to obstruct the growth of fibers.

We propose a grafting strategy (Figure 1) involving the incorporation of neural cells into highly porous polymeric matrices (i.e. macroporous hydrogel (Step 1 of Fig. 1) which display three functions converging toward promoting neural graft integration:

1. Structural: Copolymeric matrices of methacrylate polymer and collagen serve as artificial bridge substrates into lesioned areas of the brain parenchyma, and provide a support to guide tissue ingrowth and organization, glial remodelling at the interface and axonal regeneration into the pore channels (Step 1a of Fig. 1; /4/).

2. Chemical: Hydrogels allow transport of oxygen, ions and small molecules, and, by absorbing molecules from interstitial fluids, may maintain a physiological milieu with locally increased concentrations of trophic factors and other molecules relevant for regeneration/ survival.

3. Cell carriers: A prerequisite for Step 2 (Fig. 1) (i.e. placement of the neural cells within a threedimensional polymeric matrix) is the selection of a polymer system that has desired properties re- garding neural cell-polymer interactions. Neural cell attachment and axonal growth are influenced by the chemical nature of the polymer and by modifications of the polymer surface (e.g. attachment of biologically active molecules or functional chemical groups).

We studied different polymer systems for their ability to interact with neuronal cells. Embryonic neurons, free of glia and seeded at 2 $x 10^{3}$ cells $/ \mathrm{mm}^{2}$, attach preferentially to poly $(\mathrm{N}$ hydroxypropyl methacrylamide) (pHPMA) containing amide bonds, and the neural cells form stable cohesive bonds with glucosamine covalently linked to pHPMA matrices /3/. Alternative polymer systems with high hydroxyl content or containing hydrophobic fractions are not effective in promoting adhesion or fiber growth. Axonal outgrowth is promoted by polymers synthesized with collagen. Viability screening tests using an MTT tetrazolium assay /2/ demonstrated the maintenance of viability and metabolic activity of neurons seeded onto such polymer substrates.

Embryonal carcinoma-derived neural cell micro-aggregates, as model donor cells $/ 1 /$ or embryonic neurons incorporated into polymeric matrices (either by direct injection or by rehydration-entrapment) can be maintained in vitro and the cells continue to differentiate. This approach provides the basis for a new strategy in neurotransplantation: implantation of polymeric matrices seeded with donor cells (Step 3 of Fig. 3). Our current efforts are aimed toward the optimization of the microstructure and surface modifications of the matrices. We hope to generate multifunctional matrices possessing more than one type of functional group and properties suitable for promoting attachment and growth of 


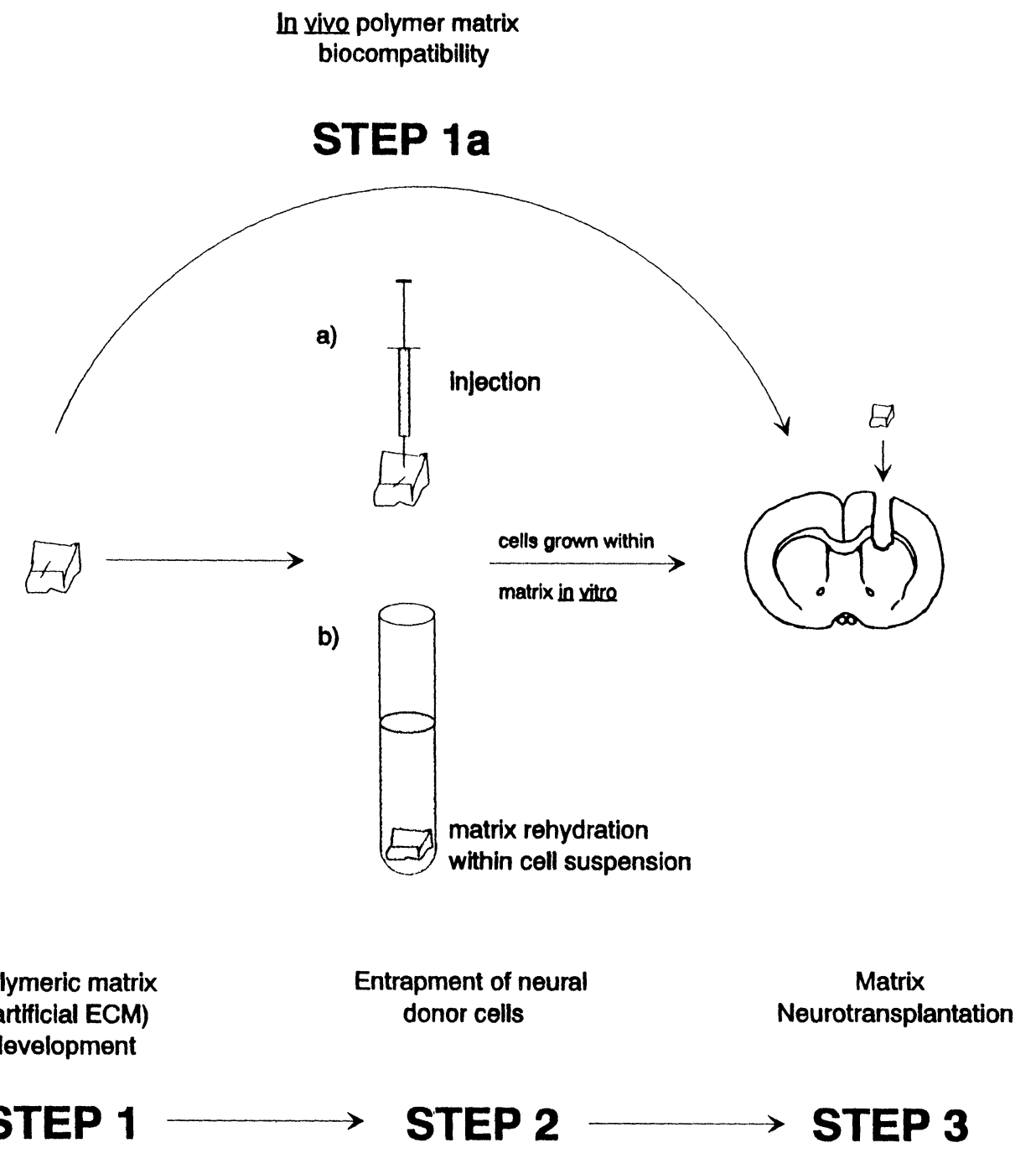

Fig. 1: Schematic representation of neural graft implantation within polymer matrices

neural grafts and regenerating host tissue.

\section{REFERENCES}

Supported by the Medical Research Council of Canada.
1. Morasutti DJ et al. Submitted 1992.

2. Mosman TJ. Immunol Meth 1983; 65: 55-63.

3. Woerly S, Maghami G, Duncan R, Subr V, Ulbrich K. Brain Res Bull 1992 (in press)

4. Woerly S, Marchand R, Lavalle C. Biomaterials 1990; 11: 97-107, 1990. 

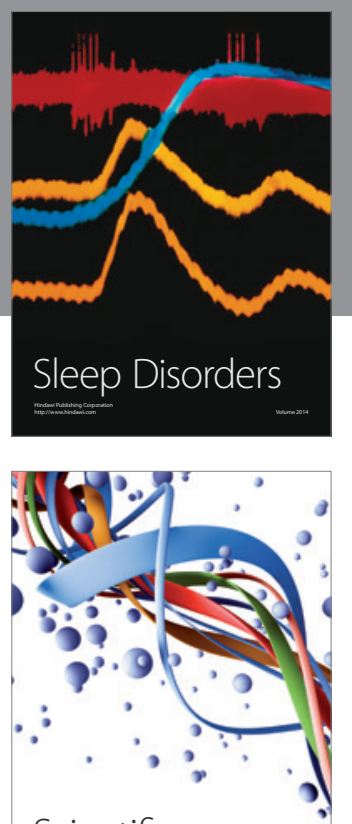

Scientifica
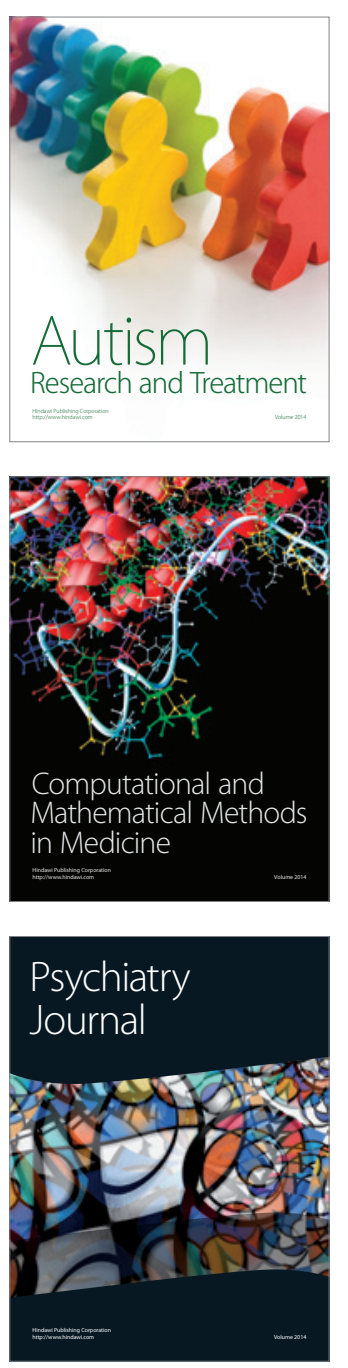
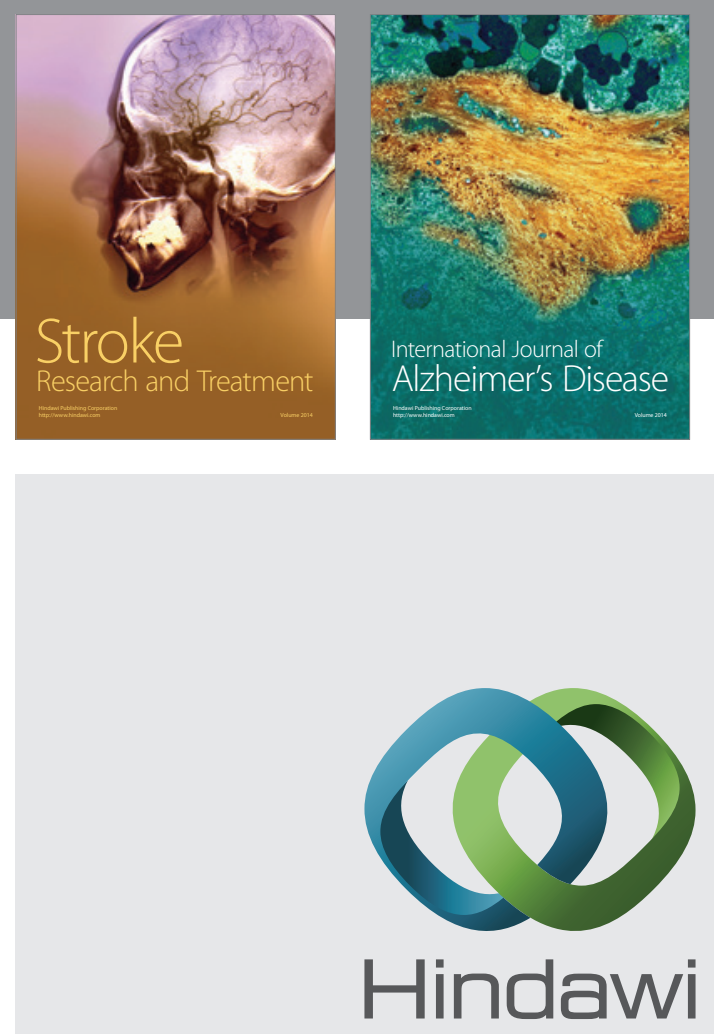

Submit your manuscripts at

http://www.hindawi.com
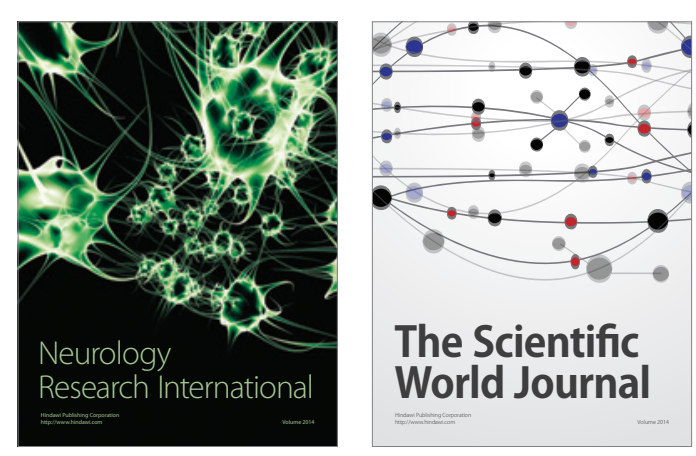

The Scientific World Journal

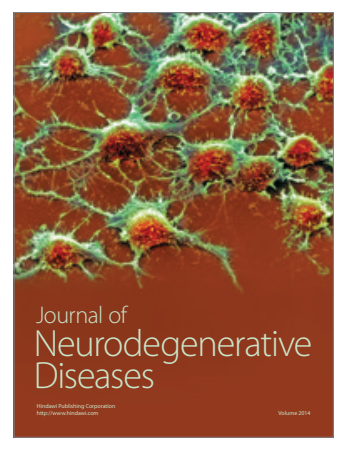

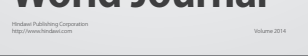

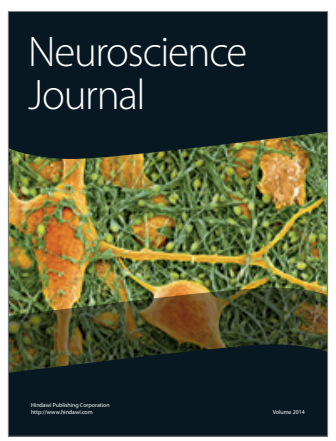

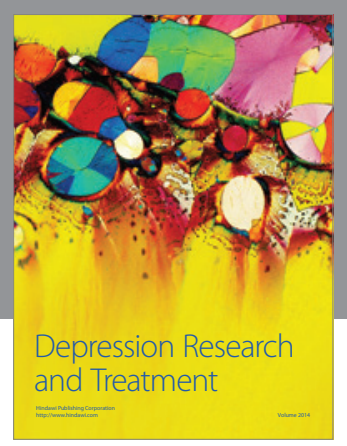
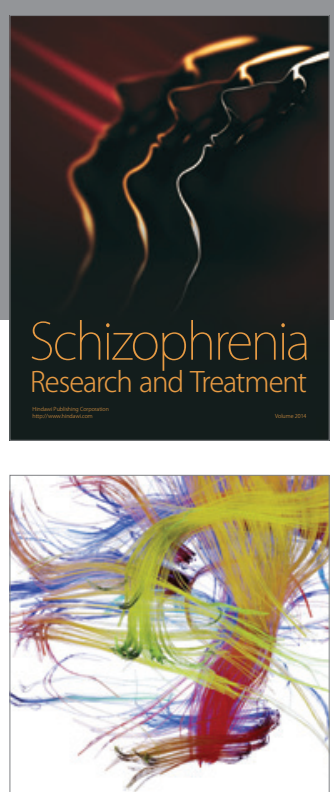

Brain Science

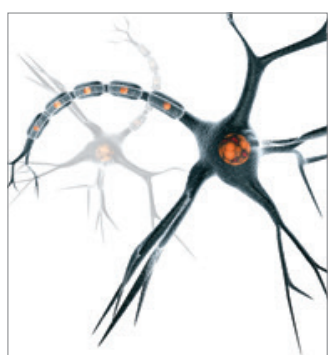

Neural Plasticity
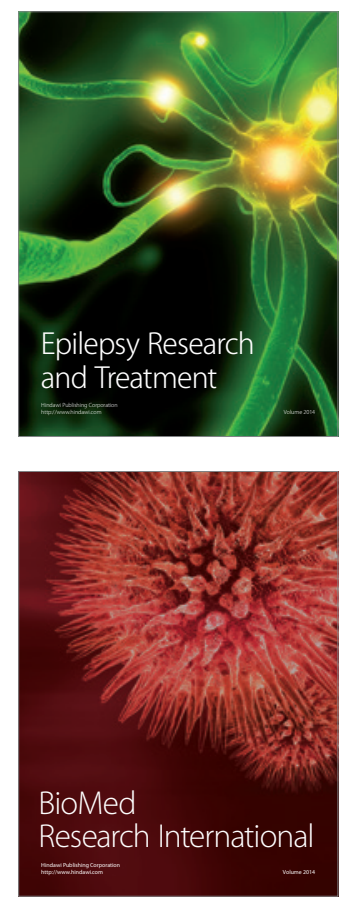

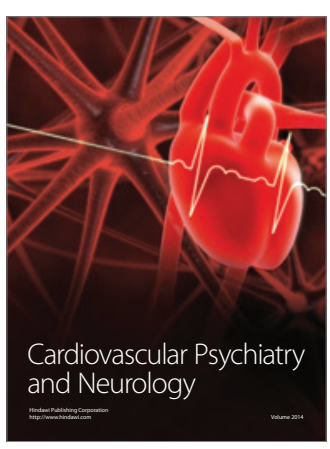

Parkinson's

Disease
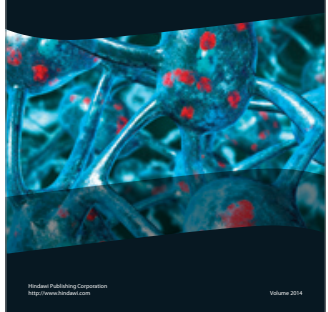